\title{
Efficient Hardware Implementation of ESPRIT-like Algorithm for Range Estimation of Chirp Spread Spectrum
}

\author{
Arjumand Yaqoob, Umar Farooq, Ghulam Abbas, and Muhammad Usman Asad
}

\begin{abstract}
Range estimation of IEEE 802.15.4a CSS, a new ESPRIT-like algorithm is presented. The conventional ranging algorithms have estimated frequency components from channel frequency response of the received signals. However, the proposed algorithm which is based on the separated multi-band chirp signals can perform range estimation by using the phase relation between sub-chirps in low and high bands. The simulation and implementation results of the proposed algorithm have a gain of about $5 \mathrm{~dB}$ and improved range error ratio of $3 \sim 5 \%$, respectively, compared to ESPRIT algorithm. The proposed algorithm is verified for hardware implementation using FPGA. The implementation results show that the computational acceleration achieved can be employed for efficient hardware implementation of a Ranging system on chip.
\end{abstract} Chip

Index Terms-Range estimation, CSS, Ranging System on

\section{INTRODUCTION}

CSS is the new IEEE Std.P802.15.4a at 2.4GHz as in [1], which uses multi-band chirp signals for data communication. Since the received chirp signals can be transformed to sinusoids by the dechirping process as in [2], super-resolution (SR) algorithms such as ESPRIT, MUSIC and matrix pencil can be employed to estimate the propagation delay of arrival paths in CSS without an additional channel frequency response estimation process as in [4][6]. Unlike general chirp signal based system, CSS transmits the same sub-chirp signal via two different bands, so the dechirped sinusoids of the received sub-chirp signal in high-band have the same frequency as those of the received sub-chirp signal in low-band, but with different phases. This phase difference is a function of the center frequency difference between low and high bands and propagation delay of arrival paths. Therefore, we can extract propagation delay information from the estimated phase difference for range estimation. However, only the first arrival path contains the propagation delay information for the direct path

Manuscript received October 14, 2010; revised July 6, 2011

Arjumand Yaqoob is with Department of Information and Communication, Hanyang University, Seoul 133-791 Republic of Korea (phone: +82-2-2220-05585; e-mail: arjumandyaqoob@gmail.com).

Umar Farooq is with Department of Electrical Engineering, College of Engineering \& Emerging Technologies, University of The Punjab Quaid-e-Azam Campus Lahore-54590 Pakistan (phone: +923217264147; e-mail: engr.umarfarooq@yahoo.com).

Ghulam Abbas is with Lyon Institute of Nanotechnology (INL), University of Lyon, CPE Lyon France (email: ghulam.abbas@cpe.fr)

Muhammad Usman Asad is with Department of Electrical Engineering, University of The Punjab Lahore-54590 Pakistan (phone: +923234399264; email: usmanasad01@hotmail.com) between transceiver. Therefore, we consider only first arrival path among multi-path in this paper.

\section{PRELIMINARY}

\section{A. Signal Model for CSS}

CSS transmits a full chirp signal which consists of four individual sub chirp signals which use either high or low band. CSS has four piconets to support simultaneous data communication for multi-users. For our convenience without losing generality, we assume that only two sub chirps in the different two bands are used in this paper. Time-frequency relation of two sub chirps in low and high band in piconetl is shown in Fig.1. The received chirp signals in Fig.1 are delayed by $\tau_{1}$ with respect to reference signal and cause constant frequency difference between the received and reference CSS signal. The starting time of reference CSS signal in Fig. 1 is decided by correlation process by which the receiver can perform coarse estimation for $\tau_{1}$. By multiplying the received CSS signal with the conjugated reference CSS signal, the dechirped sinusoids of frequency $-\mu \tau_{m}$ can be generated as in (1).

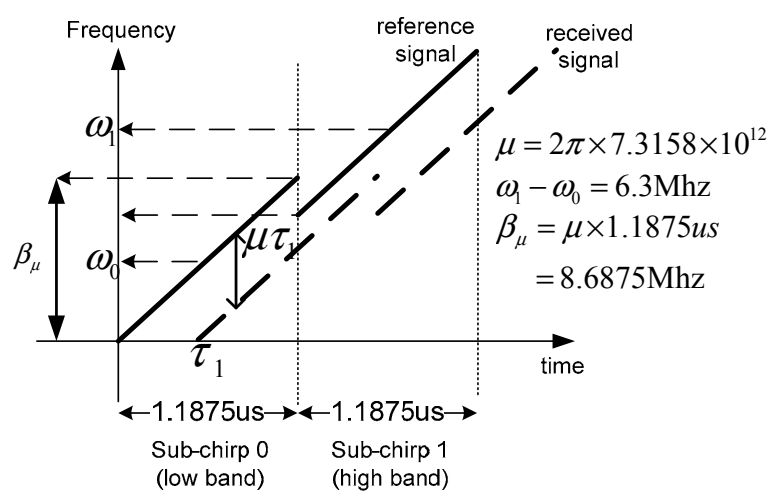

Figure 1. Time frequency relation of two sub chirp signals

In the Fig. $1, \beta_{\mu}$ is the amount of the swept frequency, other parameters are explained below (1):

$$
y_{k}(t)=\sum_{m=1}^{d} a_{m} \exp \left[j\left(-\mu \tau_{m} t-\omega_{k} \tau_{m}+\frac{\mu}{2} \tau_{m}^{2}\right)\right]+n^{\prime}(t)
$$

where $k \in\{0,2\}$ and $n^{\prime}(\mathrm{t})$ is the projection of AWGN onto the sub chirp replica. The $\omega_{k}$ is the center frequency of the $k$-th sub chirp, while $a_{m}$ and $\tau_{m}$ denotes $m$-th path's amplitude and propagation delay with respect to start time of reference signal in the receiver. We can estimate the start time of reference chirp signal which is the coarse start time of the received signal by correlation method between reference and 
received signal. $d$ is the total number of paths and constant $\mu$ defines the inclination of sub-chirp signal in time-frequency domain. As shown in (1), the frequency components of dechirped sinusoids are oriented from $\tau_{m}$. Therefore, the multipath delay of CSS can be estimated from frequency estimation.

\section{B. Conventional ESPRIT Approach}

In case of ESPRIT, the received dechirped sinusoids of (1) can be used to form a correlation matrix $R_{k}$ and EVD can be applied to remove the noise component in (1). Then, we can estimate multi-path component as following:

$$
\begin{aligned}
& \hat{\mathbf{R}}^{(0)}=\hat{\mathbf{R}}^{(1)} \Phi \text { where } \Phi=\mathbf{T}^{-1} \Lambda \mathbf{T} \\
& \text { and } \Lambda=\operatorname{diag}\left[e^{j\left(-\mu T_{s} \tau_{1}+\theta_{1}\right)}, \ldots, e^{j\left(-\mu T_{s} \tau_{m}+\theta_{m}\right)}\right]
\end{aligned}
$$

where $\mathrm{T}_{\mathrm{s}}$ is the sample duration and $\theta$ is the induced error due to AWGN noise while calculating $\Lambda . \hat{\mathbf{R}}^{(0)}$ and $\hat{\mathbf{R}}^{(1)}$ are obtained from $R_{k}$ matrix by deleting its first and last rows respectively. The above equation can be solved for eigen values $\Lambda$, and from estimated values each of the propagation delay $\tau_{\mathrm{m}}$ can be measured. The minimum value of $\ln (\Lambda) /-j \mu T_{s}$ can be selected to calculate the estimated propagation delay for direct path as following:

$$
\hat{\tau}_{1}=\frac{\min (\ln (\Lambda))}{-j \mu T_{s}}=\frac{-\mu T_{s} \tau_{1}+\theta_{1}}{-\mu T_{s}}=\tau_{1}+\frac{\theta_{1}}{-\mu T_{s}} .
$$

It is evident through above equation that the estimated $\hat{\tau}_{1}$ would be more robust to noise $n^{\prime}(\mathrm{t})$ if the divider in above equation becomes more large. If one sub-chirp is composed of $\mathrm{N}$ samples we can rewrite (3) as:

$$
\begin{gathered}
\hat{\tau}_{1}=\tau_{1}+\frac{\theta_{n}}{-\left(\frac{\beta_{\mu}}{N T_{s}}\right) T_{s}} \approx \tau_{1}+\frac{\theta_{n}}{-\left(\frac{\left|\omega_{k}-\omega_{k+1}\right| \times \alpha}{N}\right)} \approx \tau_{1}+\eta_{\text {single }} \\
\text { where } \alpha=\left|\omega_{k}-\omega_{k+1}\right| / \beta_{\mu}=6.3 / 8.6875 \approx 0.73 .
\end{gathered}
$$

Single represents the approximated propagation delay error.

\section{PROPOSED ESPRIT APPROACH}

The Proposed algorithm can estimate propagation delay $\tau_{\mathrm{m}}$ based on the phase relation between dechirped sinusoids of sub chirps in low and high bands. This phase relation can be formulated as following:

$$
y_{k, m}^{\prime}(t)=y_{k+1, m}^{\prime}(t) \times \exp \left[j\left(\left(\omega_{k}-\omega_{k+1}\right) \tau_{m}\right)\right], \text { for } k \in\{0,2\}
$$

where $y_{k, m}^{\prime}(\mathrm{t})$ and $y_{k+1, m}^{\prime}(\mathrm{t})$ are the dechirped sinusoids of $m$-th path of the noiseless form of $y_{k}(\mathrm{t})$ and $y_{k+l}(\mathrm{t})$, respectively. If we can estimate this phase $\left(\omega_{k}-\omega_{k+1}\right) \tau_{m}$ denoted by $\psi$ using ESPRIT-like algorithm, then the estimated $\tau_{m}$ would be more robust than the result from (3), since divider of $\theta_{1}$ in below equation (6) becomes more larger than that of (4):

$$
\begin{aligned}
\hat{\tau}_{m} & =\frac{\ln (\Lambda)}{-j\left(\omega_{k}-\omega_{k+1}\right)}=\frac{\left(\omega_{k}-\omega_{k+1}\right) \tau_{m}+\theta_{1}}{\omega_{k}-\omega_{k+1}}=\tau_{m}+\frac{\theta_{1}}{\omega_{k}-\omega_{k+1}} \\
& =\tau_{m}+\eta_{\text {double }} \text { where } \eta_{\text {double }} \approx \alpha \frac{\eta_{\text {single }}}{N}
\end{aligned}
$$

Double is the approximated delay error associated with ESPRIT like algorithm? In case of ESPRIT, auto-correlation and EVD is performed to separate noise space from signal space. However, the proposed algorithm doesn't use autocorrelation matrix in order to retain the phase information between dechirped sinusoids in low and high bands. Instead of auto-correlation matrix, we use data matrices $Y_{k}$ and $Y_{k+1}$ and joint SVD as following:

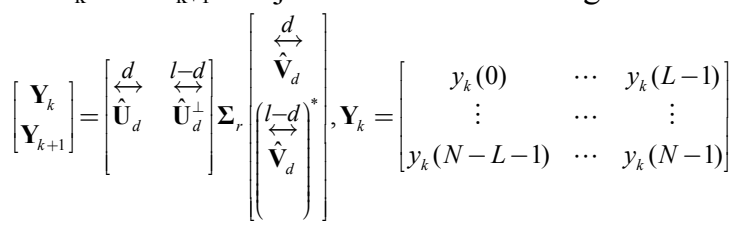

where $d$ is the number of multipath as in (1) as well as the number of the column vectors which span signal space of $\left[\mathrm{Y}_{\mathrm{k}} \mathrm{Y}_{\mathrm{k}+1}\right]^{\mathrm{T}}$. After joint SVD, we derive signal space of $\left[\mathrm{Y}_{\mathrm{k}}\right.$ $\left.\mathrm{Y}_{\mathrm{k}+1}\right]^{\mathrm{T}}$ according to the left $d$ singular values as in [4] and perform TLS-TLS approximation as following:

$$
\begin{aligned}
& \hat{\mathbf{Y}}_{k}=\prod_{\hat{\mathbf{U}}, c} \mathbf{Y}_{k} \prod_{\hat{\mathbf{V}}, r}=\hat{\mathbf{U}}_{c} \hat{\mathbf{U}}_{c}^{*} \mathbf{Y}_{k} \hat{\mathbf{V}}_{r} \hat{\mathbf{V}}_{r}^{*}, \\
& \hat{\mathbf{Y}}_{k+1}=\prod_{\hat{\mathbf{U}}, c} \mathbf{Y}_{k+1} \prod_{\hat{\mathbf{V}}, r}=\hat{\mathbf{U}}_{c} \hat{\mathbf{U}}_{c}^{*} \mathbf{Y}_{k+1} \hat{\mathbf{V}}_{r} \hat{\mathbf{V}}_{r}^{*}
\end{aligned}
$$

where $\quad \Pi \hat{\mathbf{v}}, r=\hat{\mathbf{V}}_{d} \hat{\mathbf{V}}_{d}{ }^{*}$ and $\quad \prod \hat{\mathbf{U}}, c=\hat{\mathbf{U}}_{d} \hat{\mathbf{U}}_{d}{ }^{*}$ denote the projector onto the approximated row space and column space of $\left[\mathrm{Y}_{\mathrm{k}} \mathrm{Y}_{\mathrm{k}+1}\right]^{\mathrm{T}}$, respectively. $\hat{\mathbf{Y}}_{k}$ and $\hat{\mathbf{Y}}_{k+1}$ are referred as filtered matrix in this paper. Each data matrix of size $L$ by $\mathrm{N}-\mathrm{L}$ was made without the knowledge about signal space of dimension $d$. Therefore, the filtered matrix is also of size $L$ by $N-L$. However, this filtered matrix can not be directly applied to key parts of conventional ESPRIT procedures after (10) due to its size as in [3]. Therefore, we reconstruct the received sample sequence by using the filtered_matrix as in (9) and form a matrix of size N-d by d composed of the reconstructed sequence.

$$
\begin{aligned}
& \hat{y}_{k}(m)=\sum_{x} \sum_{y} \hat{\mathbf{Y}}_{k}(x, y) \text { where } \\
& \{(x, y) \mid x+y=m \text { and } x \in\{0, \ldots, L-M-1\}, y \in\{0, \ldots, L\}\}
\end{aligned}
$$

$\hat{\mathbf{E}}_{k}$ and $\hat{\mathbf{E}}_{k+1}$ denotes the reconstructed matrix using the low and high bands of sub chirps, respectively.

$$
\hat{\mathbf{E}}=\left[\hat{\mathbf{E}}_{k} \mid \hat{\mathbf{E}}_{k+1}\right] \text { where } \hat{\mathbf{E}}_{k}=\left[\begin{array}{ccc}
\hat{y}_{k}(0) & \cdots & \hat{y}_{k}(d-1) \\
\vdots & \cdots & \vdots \\
\hat{y}_{k}(N-d-1) & \cdots & \hat{y}_{k}(N-1)
\end{array}\right]
$$

The last step below is similar to conventional ESPRIT algorithm as in [5-6]. We try to find a matrix $\mathbf{F}=\left[\begin{array}{c}\mathbf{F}_{k} \\ \mathbf{F}_{k+1}\end{array}\right]$ to 
minimize $\mathbf{V}=\left\|\left[\hat{\mathbf{E}}_{k} \mid \hat{\mathbf{E}}_{k+1}\right] \mathbf{F}\right\|_{F}^{2}$ subject to $\mathbf{F} \times \mathbf{F}=\mathbf{I}$. It is easily shown that $\mathrm{F}$ is the matrix of right singular vectors of $\left[\hat{\mathbf{E}}_{k} \mid \hat{\mathbf{E}}_{k+1}\right]$ corresponding to the $d$ smallest singular values as in [3]. The phase difference in (5) can be found as the eigen-values of $-\mathbf{F}_{k} \mathbf{F}_{k+1}{ }^{-1}$.

\section{RANGING ESTIMATION AND IMPLEMENTATION RESUlTS}

The proposed algorithm has been verified through simulations and implementation results. The simulations are performed for AWGN channel and experimental results for implementation have been drawn for measuring the distance between transceivers of CSS. The proposed algorithm is compared with single and double BW ESPRIT as shown in Fig.2 and 3. Actually the single BW ESPRIT is based on one sub chirp of CSS, while double BW ESPRIT is based on the continuous chirp signal which uses full bandwidth in contrast with CSS signal.

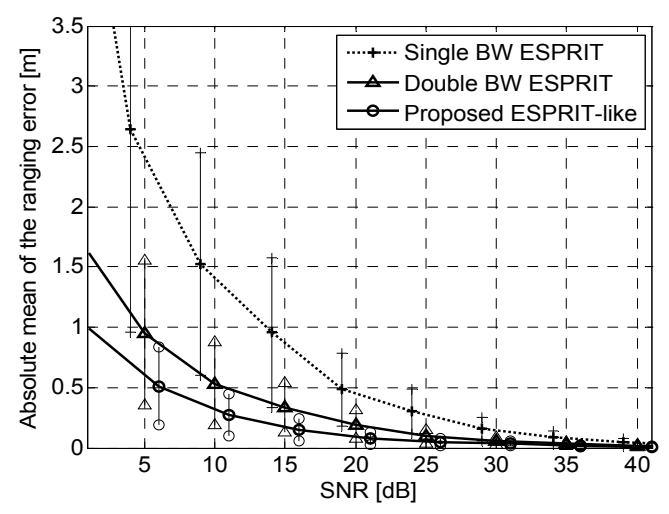

Figure 2. The simulation results in AWGN channel

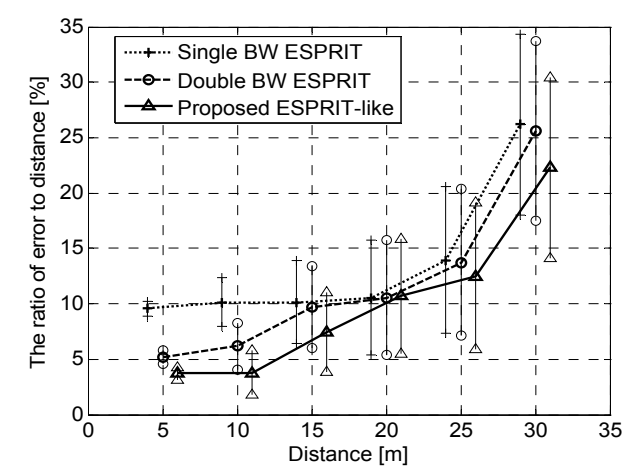

Figure 3. Experimental results in indoor environment

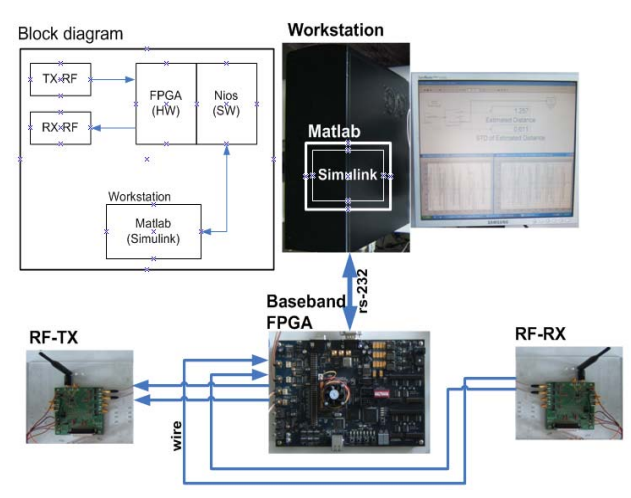

Figure 4. Ranging system block diagram
In Fig.2, the mean and STD of ranging errors are compared and the range errors are mapped according to measured distances between transceivers in indoor geo location in Fig.3.

In Fig.2and 3 the vertical line shows the STD of the range error while the centre of vertical line is the mean value for measurements. The simulation results can embark the superiority of the algorithm over ESPRIT by achieving the SNR gain of about $5 \mathrm{~dB}$, compared to the double BW ESPRIT. Similarly for the ratio of error to measured distances as mapped in Fig.3 shows less error ratio about 3 5\% for proposed algorithm as compared to double BW ESPRIT.

In Fig.4 the block diagram of the implemented system used for ranging estimation is shown. The system comprises of a base band FPGA board by Altera Startix-II EP2S180 and two RF transceiver MAX2830 EV KITs. This implemented system can transmit and process various chirp signals for range estimation. The chirp signal is stored in FPGA board and transmitted by RF module, while the received chirp signal is detected for range estimation by the hardware part of the board. NIOSII core of the board control the hardware part and passed the received samples to the PC whereas the high resolution algorithm the ESPRIT like algorithm has been implemented in part by employing the hardware and software blocks implemented using Simulink and the Singular value decomposition (SVD) part in hardware on FPGA.

\section{CONCLUSIONS}

In this paper, we proposed and implemented a new ESPRIT-like algorithm for efficient range estimation. The algorithm can be used for range estimation using CSS signal. This algorithm utilized the CSS sub-chirp signals in different two bands more efficiently than ESPRIT and the hardware implementation verifies this concept to be used in efficient ranging systems. Therefore, we expect that this algorithm can be applied to ranging system which uses multi-band chirp signals. In this regard an efficient ranging system can be built using CSS.

\section{ACKNOWLEDGMENT}

This research was supported by the MIC (Ministry of Information and Communication), Korea, under the ITRC (Information Technology Research Center) support program supervised by the IITA (Institute of Information Technology Advancement)(IITA-2008-(C1019-0701-0019)).

\section{REFERENCES}

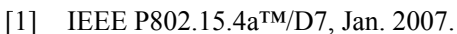

[2] Djuric P.M, Kay S.M, "Parameter estimation of chirp signals," IEEE Transactions Acoustics Speech. and Signal Processing, Vol.38,pp.2118-2126, Dec.1990.

[3] A.J. van der Vaen, E.F, Deprettite, and A.L. Swindlehurst, "Subspace based signal analysis using singular value decomposition," Proc. IEEE vol.81, pp.1227-1308, Sep. 1993.

[4] Xinrong Li, Pahlavan K, "Super-resolution TOA estimation with diversity for indoor geo-location," IEEE Transactions Wireless Communications, vol.3, pp .224-234, Jan. 2004.

[5] Dharmadial N, Adve. R, Farha R M, "Multipath delay estimation using matrix pencil," Proc. WCNC., vol.1, pp.632-635, Mar. 2003. 
[6] Paulraj A, Roy R, And Kailath T, "A subspace rotation approach to signal parameter estimation,” Proc. IEEE, vol.74, no. 7, July 1986.

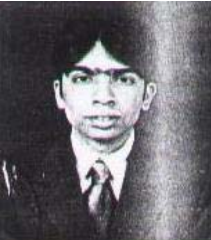

Arjumand Yaqoob, was born on 19th April 1981 Lahore Pakistan. He holds a Bachelor's degree in Electrical Engineering (2004), and a Master's degree in Electrical Engineering (2008), from University of Engineering \& Technology Lahore. He has more than five years of experience working in design of digital systems. His particular interests include SOC design and hardware optimizations and implementations of communication algorithms.

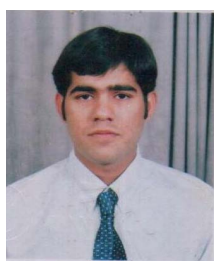

Umar Farooq, did his B.Sc. and M.Sc. both in Electrical Engineering from University of Engineering \& Technology Lahore in 2004 and 2010 respectively. $\mathrm{He}$ is currently with the Department of Electrical Engineering, University of The Punjab Lahore. His research interests include the application of intelligent techniques to problems in control engineering, robotics and power electronics.

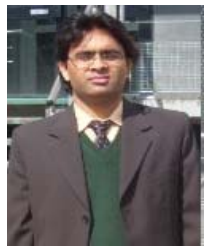

Ghulam Abbas, received the B.Sc. degree in Electrical Engineering from University of Engineering \& Technology Lahore in 2004. Currently he is working towards the $\mathrm{PhD}$ degree in Electrical Engineering at Lyon Institute of Nanotechnology (INL), University of Lyon, CPE Lyon, France. His current research interests include the design of classical and intelligent controllers for high frequency dc-dc converters

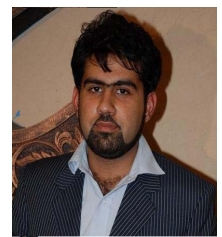

Muhammad Usman Asad, received the B.Sc. degree in Electrical Engineering from University of The Punjab Lahore in 2011. While his stay at University of The Punjab Lahore, he procured fame to his university by wining various contests related to circuit design and robotics; organized by prestigious engineering institutions of Pakistan. He is also the receipt of gold medal and silver medal awards for oral presentation of his research papers at $24^{\text {th }}$ and $26^{\text {th }}$ IEEEP International Multi-topic Symposium held at PC, Karachi. His research interests include circuit and systems, fuzzy systems, neural networks, robotics and mechatronics. 